\title{
Orbital frustration at the origin of the magnetic behavior in $\mathrm{LiNiO}_{2}$
}

\author{
F. Reynaud ${ }^{1}$, D. Mertz ${ }^{1}$, F. Celestini ${ }^{1}$, J.-M. Debierre ${ }^{1}$, A. M. Ghorayeb ${ }^{1}$, P. Simon ${ }^{2}$, A. Stepanov ${ }^{1}$, \\ J. Voiron ${ }^{3}$, and C. Delmas ${ }^{4}$ \\ ${ }^{1}$ Laboratoire Matériaux et Microélectronique de Provence, Case 151, Université d'Aix-Marseille III and CNRS, \\ 13397 Marseille Cedex 20, France \\ ${ }^{2}$ Centre de Recherche sur les Matériaux à Hautes Températures, CNRS, 45071 Orléans Cedex 2, France \\ ${ }^{3}$ Laboratoire Louis Néel, CNRS, Boîte Postale 166, 38042 Grenoble Cedex 9, France \\ ${ }^{4}$ Institut de Chimie de la Matière Condensée de Bordeaux, Ecole Nationale Supérieure de Chimie et Physique de Bordeaux \\ and CNRS, 33608 Pessac Cedex, France
}

(february 27, 2001)

\begin{abstract}
We report on the ESR, magnetization and magnetic susceptibility measurements performed over a large temperature range, from 1.5 to $750 \mathrm{~K}$, on high-quality stoichiometric $\mathrm{LiNiO}_{2}$. We find that this compound displays two distinct temperature regions where its magnetic behavior is anomalous. With the help of a statistical model based on the Kugel'-Khomskii Hamiltonian, we show that below $T_{o f} \simeq 400 \mathrm{~K}$, an orbitally-frustrated state characteristic of the triangular lattice is established. This then gives a solution to the long-standing controversial problem of the magnetic behavior in $\mathrm{LiNiO}_{2}$.

PACS numbers: 75.40.Cx, 76.30.-v, 75.30.Et, 75.40.Mg
\end{abstract}

The problem of orbital degeneracy (OD) in transitionmetal oxides has attracted considerable interest recently, due to its drastic effect on the magnetic, elastic, transport and other properties of these compounds [1]. Among the most studied oxides exhibiting OD are manganites, vanadates, some titanates and other systems. Here we discuss a new interesting aspect of OD: the frustration of degenerate orbitals, as applied to lithium nickel oxide.

Since $\mathrm{LiNiO}_{2}$ was first studied as an ionic conductor [2], the magnetic properties of this layered compound have been reported in numerous publications but are still the subject of considerable controversy. Quantum spin liquid [3], spin glass [4] 7], frustrated antiferromagnetism [4.,8 10], quantum disordered state without a spin gap 111] and other scenarios [12 20] have been claimed to be responsible for the magnetism of this material. Actually, interest in this compound stems essentially from two facts. First, following the suggestion by Anderson 21] that a resonating valence bond ground state may exist in a $\mathrm{S}=1 / 2$ Heisenberg triangular-lattice antiferromagnet, $\mathrm{LiNiO}_{2}$ was proposed by Hirakawa et al. [3] as a potential physical realization of such a system. The second important feature, overlooked in earlier studies, is the unusual electronic state of $\mathrm{Ni}^{3+}$ ions. The strong crystal field acting on the nickel ions brings them in the low-spin state $\left(t_{2 g}^{6}, e_{g}^{1} ; S=1 / 2\right)$, with the two $e_{g}$ levels being degenerate. Clearly, OD should play a crucial role in the magnetic properties of this compound 11,22,23. However, no direct confirmation of this hypothesis exists yet.

In this paper we propose a novel scenario for $\mathrm{LiNiO}_{2}$ based on an interplay between frustration on a triangular lattice and $\mathrm{OD}$ of $\mathrm{Ni}^{3+}$ ions. We show experimentally that an anomalous magnetic behavior occurs in two different temperature regions, which we interpret as the consequence of two distinct energy scales present in the system. The first one, of the order of hundreds of Kelvin, is related to the antiferro-orbital coupling between $\mathrm{Ni}^{3+}$ ions and leads to a frustrated distribution of $\mathrm{Ni}^{3+}$ orbitals. The second one, of the order of tens of Kelvin, is due to the spin-spin interactions between electrons in these frustrated $\mathrm{Ni}^{3+}$ orbitals, and drives the very unusual magnetic behavior at low temperatures. Our proposed scenario, which we confirm through a direct comparison between experimental data and simulation results, gives a coherent and natural explanation of this behavior.

High-quality powder samples of stoichiometric $\mathrm{LiNiO}_{2}$ have been prepared following the procedure described by Rougier et al. [24. We have studied these samples by $\mathrm{X}$-band $(\nu=9.45 \mathrm{GHz})$ electron spin resonance (ESR), magnetization (up to $15 \mathrm{~T}$ ) and dc-susceptibility measurements over a wide temperature range, from 1.5 to $750 \mathrm{~K}$.

The ESR spectra have a symmetric shape at all temperatures and show no trace of any particular feature that would be representative of ferrimagnetic cluster formation or of any anisotropy. This is a first but clear indication that the samples studied in this work are homogeneous and of good quality. We show in Fig. 1 the $T$ dependence of the ESR linewidth, $\Delta H$ (half width at half maximum), of a typical sample of $\mathrm{LiNiO}_{2}$ in the low- $T$ regime. A huge increase of $\Delta H$ is noted when the temperature decreases below $50 \mathrm{~K}$. This temperature, to which we shall refer below as $T_{m}$, quite naturally gives the scale of the magnetic interaction in $\mathrm{LiNiO}_{2}$. Below $T \simeq 10 \mathrm{~K}$, $\Delta H$ tends to a saturation value of about $0.5 \mathrm{~T}$. The inset of Fig. 1 shows the inverse of the spin susceptibility, $\chi^{-1}(T)$, deduced from the ESR spectra. As already reported earlier [5], $\chi(T)$ follows a Curie-Weiss behavior, $\chi=C /(T-\theta)$, down to about $80 \mathrm{~K}$, with $\theta \simeq 35 \pm 5 \mathrm{~K}$. This low value of $\theta$ confirms that the sample studied is so close to stoichiometry that we may consider it as pure 
$\mathrm{LiNiO}_{2}$,5.11]. Bearing in mind that $\theta$ represents the average sum of exchange interactions on an atomic site, the positive sign of $\theta$ implies that the dominant interactions are ferromagnetic (FM). However, the line broadening observed in Fig. 1 is rather indicative of strong antiferromagnetic (AF) fluctuations. Line narrowing would be expected if the interactions were purely ferromagnetic. Futhermore, between $13 \mathrm{~K}$ and $50 \mathrm{~K}, \Delta H$ can be fitted to the empirical formula $\Delta H=A T^{-p}$ with $p=2.65$. Such an exponent value is known to be characteristic of AF materials having a strong 2D character [25]. Another important point is the tendency of the linewidth to saturate below $10 \mathrm{~K}$. This constitutes strong evidence that the AF correlations do not propagate any longer below this temperature.

Fig. 2 shows the magnetic moment, $M$, as a function of magnetic field, $H$. The curves deviate from linearity below $T_{m} \simeq 50 \mathrm{~K}$, the same temperature below which the ESR linewidth was seen to markedly increase. At low temperatures, saturation is almost reached in fields of $15 \mathrm{~T}$ and, furthermore, below about $10 \mathrm{~K}$, temperature has hardly any effect on magnetization. Although the initial susceptibility increases with decreasing temperature in a manner reminiscent of a FM behavior, the $M(H)$ curve at the lowest temperature remains below what would be given by a Brillouin function. This confirms the existence of rather strong AF interactions along with ferromagnetism.

The second anomalous aspect of $\mathrm{LiNiO}_{2}$ is found at high temperatures and plays a crucial role in understanding the magnetic properties of this compound. Fig. 3a shows the results of the dc-susceptibility measurements, plotted in the form $(T+10) \chi(T)$ between $300 \mathrm{~K}$ and $750 \mathrm{~K}$. The curve presents a weak anomaly at around $400 \mathrm{~K}$. At higher temperatures, $\theta$, which was found to be around $35 \mathrm{~K}$ from the low- $T$ measurements, decreases to finally settle at the negative value, $\theta=-10 \pm 5 \mathrm{~K}$, for $T \geqslant 600 \mathrm{~K}$. The inset of Fig. 3a details the variation of $\theta(T)$, as deduced from $\chi(T)$. Such a drastic change in $\theta$ is quite unusual and gives strong evidence for a concomitant modification of the $\mathrm{Ni}^{3+}$ orbital distribution in the $\mathrm{NiO}_{2}$ layers. Additional support for this argument comes from the ESR data. Fig. $3 \mathrm{~b}$ presents the $T$-dependence of $\Delta H$, between $300 \mathrm{~K}$ and $625 \mathrm{~K}$. The divergence of $\Delta H$ above room temperature is quite in line with what is expected when, due to changes in the $\mathrm{Ni}^{3+}$ orbital distribution, strong fluctuations occur.

In order to explain this extremely rich and unusual magnetic behavior, we use the Kugel'-Khomskii (KK) spin-orbital model [1]. Derived from the two-band Hubbard Hamiltonian in the limit of a large Coulomb repulsion, this model has proven its efficiency to describe the interplay between magnetic and orbital ordering [26]. Here we rewrite the KK Hamiltonian in the following form :
$H=\sum_{<i, j>}\left\{-J^{o} \tau_{i} \tau_{j}-\mathbf{s}_{i} \mathbf{s}_{j}\left[J_{s o} \delta\left(\tau_{i} \tau_{j}-1\right)+J_{d o} \delta\left(\tau_{i} \tau_{j}+1\right)\right]\right\}$

where the sum runs over nearest neighbor (NN) atoms and $\delta\left(\tau_{i} \tau_{j} \pm 1\right)$ is equal to 1 if $\tau_{i} \tau_{j} \pm 1=0$ and to 0 otherwise. As usual, $\mathbf{s}_{i}$ is the spin on atomic site $i$ while the pseudo-spin, $\tau_{i}$, is introduced to describe the orbital occupancy. The values $\tau_{i}=+1$ and $\tau_{i}=-1$ respectively correspond to the two degenerate $e_{g}$ orbitals of the $\mathrm{Ni}^{3+}$ ion, $\left|x^{2}-y^{2}\right\rangle$ and $\left|3 z^{2}-r^{2}\right\rangle$. In the first term, $J^{o}$ is the orbital coupling constant. $J_{\text {so }}$ and $J_{d o}$ are the magnetic exchange constants between NN, respectively with the same and with a different orbital occupancy. The spinorbital model is here taken in its simplest form. First, we consider $\mathbf{s}_{i}$ as classical Ising spins. As a consequence, possible quantum effects at low temperatures cannot be described. Second, we do not distinguish between different relative orientations of the same orbital in the same site, as it could be done using a Potts model. Finally, we assume that the orbital coupling between two ions with the same orbital $\tau_{i}=\tau_{j}=1$ is the same as the one between two ions with $\tau_{i}=\tau_{j}=-1$. These are rather crude approximations but we shall see that this minimal model is apparently able to capture the essential physics of the system, at least for temperatures greater than $\simeq 5 \mathrm{~K}$.

Recently, van den Bossche, Zhang and Mila 23] have suggested that the $S U(4)$ symmetry $\left(J_{s o}=J_{d o}=J^{o}\right)$ could be at the origin of the magnetic properties of $\mathrm{LiNiO}_{2}$. Our high-temperature experimental data presented above suggest that $\left|J^{o}\right|>>\left|J_{s o}\right|,\left|J_{d o}\right|$. Therefore, we are here testing the relevance of the alternative $S U(2) * S U(2)$ symmetry in which the magnetic and orbital interactions have different orders of magnitude.

Concerning the sign of the orbital coupling, a ferroorbital constant $\left(J^{o}>0\right)$ would lead to a structural ferrodistortive phase transition. This possibility is completely ruled out by the available X-ray data [27] so that we have to consider an antiferro-orbital coupling constant $\left(J^{o}<0\right)$. Since the $\mathrm{Ni}^{3+}$ ions form a triangular arrangement, we then expect orbital frustration to occur below a temperature $T_{\text {of }}\left(T_{o f}=(3 / 2)\left|J^{o}\right| / k_{B}\right.$, using the mean-field approximation) in a manner similar to the well-known AF-spin-frustration phenomenon. From the experimental results shown in Fig. 3, $T_{\text {of }} \simeq 400 \mathrm{~K}$, which gives $\left|J^{o}\right| / k_{B} \simeq 270 \mathrm{~K}$. The orbitally-frustrated state may be described as a Wannier state (WS) [28]. A particular property of a WS is that three NN cannot have the same orbital occupancy. This is illustrated in Fig. 4 (a and b) where we represent typical orbital distributions above and below $T_{o f}$. It is evident that at high temperatures the probability to find a link between NN with a different orbital occupancy is $1 / 2$ and that this probability is $2 / 3$ in the WS. The mean-field values of $\theta$ are then given by:

$$
\theta_{h t}=\left(3 J_{d o}+3 J_{s o}\right) / k_{B} \text { and } \theta_{w s}=\left(4 J_{d o}+2 J_{s o}\right) / k_{B}
$$


where $\theta_{h t}$ and $\theta_{w s}$ denote the values in the hightemperature state and in the WS, respectively. The assumption of AF-orbital coupling, together with the experimentally inferred $\theta_{h t}=-10 \pm 5 \mathrm{~K}$ and $\theta_{w s}=35 \pm 5$ $\mathrm{K}$, allows us to determine the two magnetic coupling constants, $J_{d o}$ and $J_{s o}$. We find a $F M$ exchange between $N N$ with different orbitals $\left(J_{d o} / k_{B}=18 \pm 6 \mathrm{~K}\right)$ and an $A F$ one between $N N$ with similar orbitals $\left(J_{s o} / k_{B}=-20 \pm 6 \mathrm{~K}\right)$. It is interesting to note that this simple treatment of Hamiltonian (1) is sufficient for the qualitative understanding of the magnetic behavior of $\mathrm{LiNiO}_{2}$ in a large temperature interval $\left(T>T_{m}\right)$. It can also be shown that these values of $J_{d o}$ and $J_{s o}$ constitute the only combination of magnetic coupling constants that ensures the absence of a trivial long-range order, as indicated by the saturation of $\Delta H(T)$ at low temperatures (Fig. 1).

To test the ability of the model to describe the lowtemperature magnetic properties, we have performed Monte-Carlo (MC) simulations. The simulated system contains $N_{i}=36 \times 36$ coplanar ions and the classical Metropolis algorithm 29] is used. At each step both a spin and a pseudo-spin are chosen randomly and attempts to flip them are performed independently. A MC step consists of $N_{i}$ such attempts. After a first run of $2 \times 10^{4} \mathrm{MC}$ steps to ensure equilibrium, the magnetization and susceptibility are computed from uncorrelated configurations recorded during a second run of $5 \times 10^{4}$ MC steps. The best fit to the experimental results is obtained for $J_{d o} / k_{B}=21.0 \mathrm{~K}$ and $J_{s o} / k_{B}=-22.5 \mathrm{~K}$. Considering the simplicity of the model, the slight difference between these values and those deduced experimentally is not surprising. The good overall agreement found with the experimental data is rather satisfactory (Figs. 1 and 2) and allows us to interpret the magnetic behavior at intermediate and low temperatures. In the range $T_{m}<T<T_{o f}$, the magnetic state is a paramagnetic one with $\theta>0$. There are two thirds of NN pairs with different orbitals and hence a majority of FM exchanges $\left(J_{d o}>0\right)$ so that the system behaves as if approaching a FM transition. Since $\left|J_{s o}\right|>\left|J_{d o}\right|$, at lower temperatures the links between the $\mathrm{NN}$ having identical orbitals are preferably satisfied (see Fig. 4c), inducing AF-like fluctuations because $J_{s o}<0$. This situation finally leads to a magnetically frustrated state in $\mathrm{LiNiO}_{2}$ at low temperatures.

To conclude, an important outcome of our experimental results is that there are two distinct energy scales characteristic of the magnetism of $\mathrm{LiNiO}_{2}$, which respectively correspond to the antiferro-orbital coupling between $\mathrm{Ni}^{3+}$ ions in the $\mathrm{NiO}_{2}$ layers and to their spin-spin interactions. The adjunction of two facts, orbital degeneracy of the $\mathrm{Ni}^{3+}$ ions, and their triangular arrangement, leads to the build-up of a Wannier orbitally-frustrated state below $T_{\text {of }} \simeq 400 \mathrm{~K}$. This uncommon orbital state is at the origin of the observed low- $T$ complex magnetic behavior of $\mathrm{LiNiO}_{2}$.

We would like to thank D. I. Khomskii, Y. Ksari, F. Mila and F.-C. Zhang for fruitful discussions and V. A.
Pashchenko for assistance in the magnetic measurements.

[1] K. I. Kugel' and D. I. Khomskii, Sov. Phys. - JETP 37, 725 (1973); Usp. Fiz. Nauk 136, 621 (1982) [Sov. Phys. - Usp. 25, 231 (1982)].

[2] J.B. Goodenough, D. G. Wickham and W. J. Croft, J. Phys. Chem. Sol. 5, 107 (1958).

[3] K. Hirakawa, H. Kadowaki and K. Ubukoshi, J. Phys. Soc. Jpn 54, 3526 (1985); K. Hirakawa et al., J. Phys. Soc. Jpn 59, 3081 (1990) and references therein.

[4] J. N. Reimers et al., J. Sol. St. Chem. 102, 542 (1993).

[5] K. Yamaura et al., J. Sol. St. Chem. 127, 109 (1996).

[6] M. Corti et al., J. Appl. Phys. 79, 6621 (1996).

[7] A. Bajpai and A. Banerjee, Phys. Rev. B 55, 12439 (1997).

[8] H. Yoshizawa et al., J. Phys. Soc. Jpn 59, 2631 (1990); K. Hirota, Y. Nakazawa and M. Ishikawa, J. Mag. Mag. Mat. 90-91, 279 (1990); J. Phys. : Cond. Mat. 3, 4721 (1991).

[9] A. Rougier, C. Delmas and G. Chouteau, J. Phys. Chem. Sol. 57, 1101 (1996).

[10] A.-L. Barra et al., J. Mag. Mag. Mat. 177-181, 783 (1998).

[11] Y. Kitaoka et al., J. Phys. Soc. Jpn 67, 3703 (1998).

[12] J. P. Kemp, P. A. Cox and J. W. Hodby, J. Phys. : Cond. Mat. 2, 6699 (1990).

[13] P. Ganguly et al., Phys. Rev. B 46, 11595 (1992).

[14] R. Stoyanova, E. Zhecheva and C. Friebel, J. Phys. Chem. Sol. 54, 9 (1993).

[15] M. Rosenberg et al., J. Appl. Phys. 75, 6813 (1994).

[16] C. B. Azzoni et al., Phys. Rev. B 53, 703 (1996).

[17] A.-L. Barra et al., Eur. Phys. J. B 7, 551 (1999).

[18] D. Mertz et al., Phys. Rev. B 61, 1240 (2000).

[19] F. Reynaud et al., Eur. Phys. J. B 14, 83 (2000).

[20] M. D. Núñez-Regueiro et al., Eur. Phys. J. B 16, 37 (2000).

[21] P. W. Anderson, Mater. Res. Bull. 8, 153 (1973).

[22] L. F. Feiner, A. M. Oleś and J. Zaanen, Phys. Rev. Lett. 14, 2799 (1997).

[23] M. van den Bossche, F.-C. Zhang and F. Mila, Eur. Phys. J. B 17, 367 (2000).

[24] A. Rougier, P. Gravereau and C. Delmas, J. Electrochem. Soc. 14, 1168 (1996).

[25] H. W. de Wijn et al., Sol. St. Commun. 11, 803 (1972).

[26] See, for example, S. K. Pati, R. R. P. Singh and D. I. Khomskii, Phys. Rev. Lett. 81, 5406 (1998); G. Santoro et al. Phys. Rev. Lett. 83, 3065 (1999) and references therein.

[27] A. Rougier, C. Delmas and A. V. Chadwick, Sol. St. Commun. 94, 123 (1995).

[28] G. H. Wannier, Phys. Rev. 79, 357 (1950); Phys. Rev. B 7, 5017 (1973).

[29] N. Metropolis et al., J. Chem. Phys. 21, 1087 (1953). 


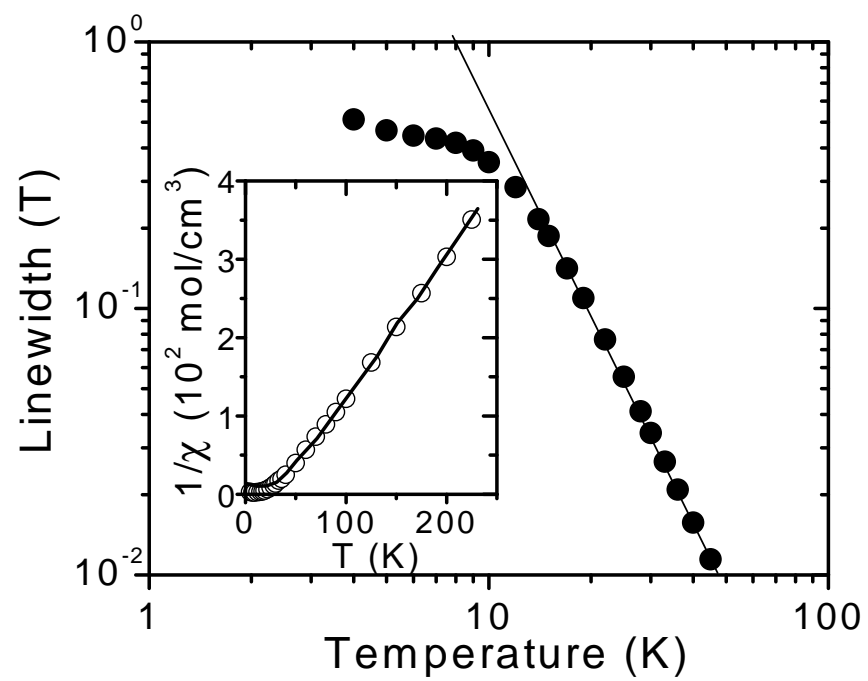

FIG. 1. Temperature dependence of the ESR linewidth, $\Delta H$. The straight line is a fit to the law $\Delta H=A T^{-p}$ with $p=2.65$. The inset shows the temperature dependence of the inverse of the spin susceptibility, $1 / \chi$, as deduced from the ESR spectra. Circles and continuous line respectively represent experimental data and numerical simulations.

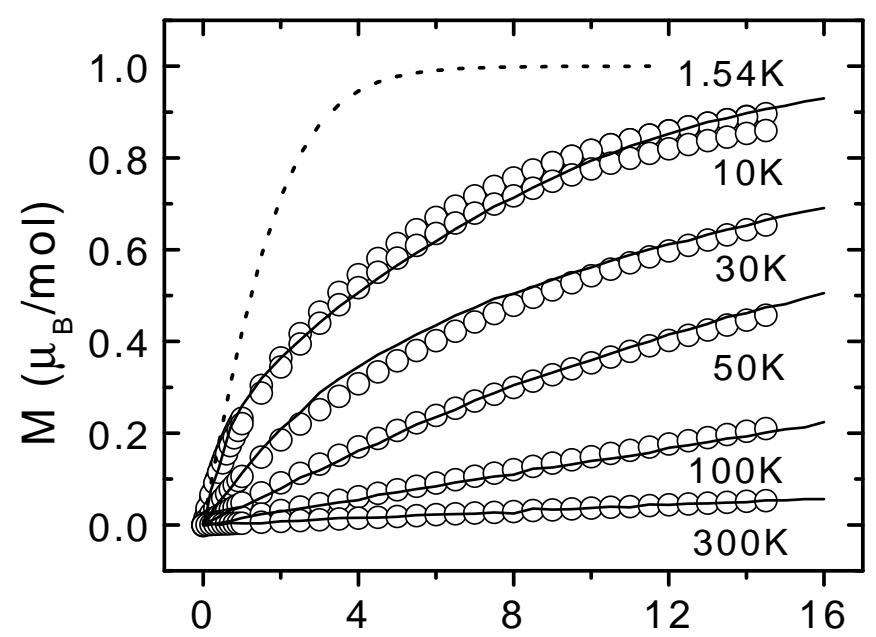

FIG. 2. Magnetic moment, $M$, as a function of magnetic field, $H$, up to $15 \mathrm{~T}$. Circles: experimental data between $300 \mathrm{~K}$ and $1.54 \mathrm{~K}$; continuous curves: simulation results; dotted curve: $M(H)$ calculated at $1.5 \mathrm{~K}$ using the Brillouin function. a)

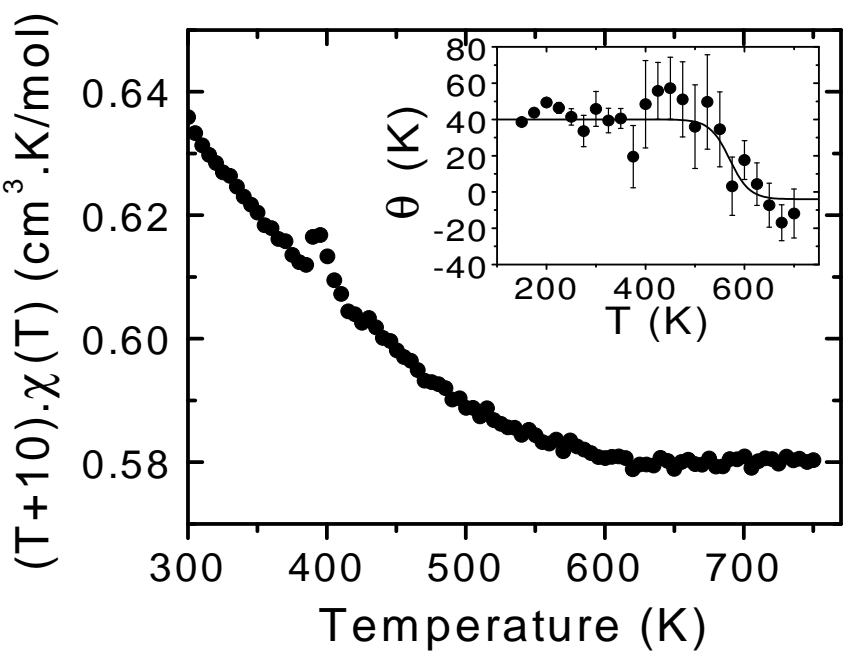

b)

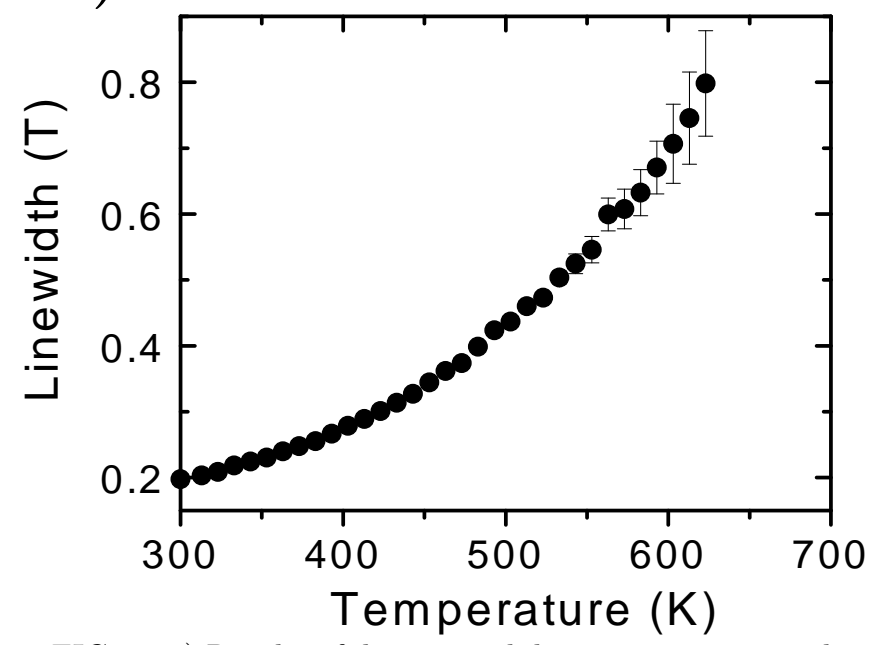

FIG. 3. a) Results of dc-susceptibility measurements, plotted in the form $(T+10) \chi(T)$. Note the plateau for $T>600 \mathrm{~K}$. The inset shows the variation of $\theta$ with temperature; the line is a guide to the eye. b) Temperature dependence of $\Delta H$ between $300 \mathrm{~K}$ and $625 \mathrm{~K}$. 
a)

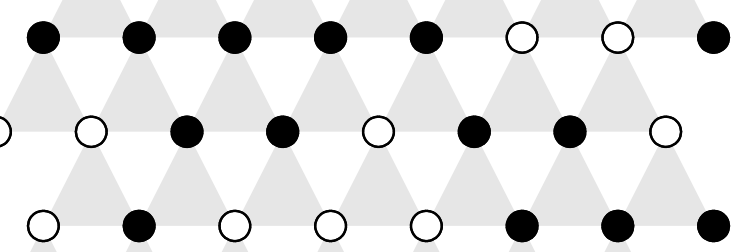

00000000

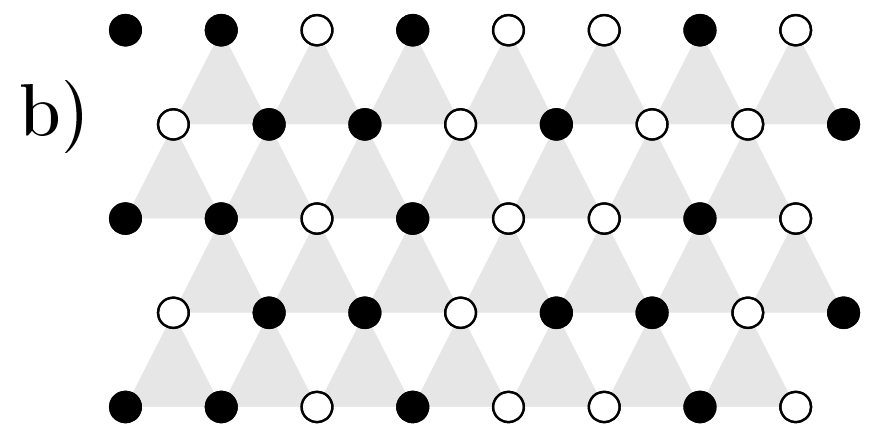

c)

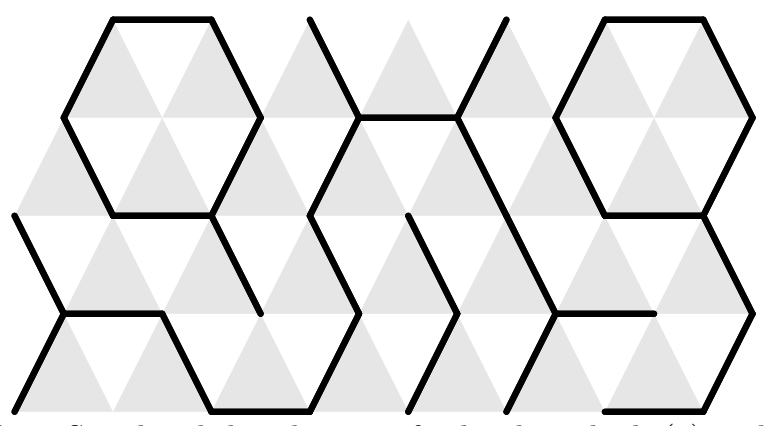

FIG. 4. Simulated distribution of orbitals at high (a) and low (b) temperatures. Filled and open circles distinguish between the two types of orbitals. c) Satisfied FM links in the low- $\mathrm{T}$ Wannier state represented in (b). 